\title{
An Information Sharing Platform for Concurrent Engineering
}

\author{
T.I.A. Ellis, A. Molina, R.I.M. Young, R. Bell \\ Manufacturing Engineering Department, Loughborough University \\ of Technology, Loughborough, Leicestershire, LE11 3TU \\ Email: r.i.young@lut.ac.uk \\ Telephone: +44 (1509) 263171 ext.2920 \\ Fax: +44 (1509) 267725
}

\begin{abstract}
Research is currently being undertaken by several research laboratories into the computer aided support of simultaneous engineering. These support systems aim to assist organisations that have adopted the simultaneous engineering philosophy by providing consistent sources of product and manufacturing information, and by providing access to a diversity of applications that support decision making.

This paper reports on one such system, the Model Oriented Simultaneous Engineering System (MOSES), and the methodology used for its development. The structure of the system is de scribed together with an outline of the novel research work that is contributing to particular elements of the system. The complexity of co-ordinating and structuring the design and development of such CAE systems necessitates the use of formal methods for their representation. A CAE Reference Model has been developed to guide the development of MOSES. The nature of the reference model is discussed.
\end{abstract}

\section{Keywords}

Concurrent Engineering, Product Model, Manufacturing Model, Reference Model

\section{INTRODUCTION}

The goals of Simultaneous Engineering (SE) are regularly espoused as being to produce products better, cheaper and faster. SE is seen as being largely dependent on increasing the consideration of life cycle activities in the early design stages [Nevins and Whitney 1989]. This has been achieved by the implementation and management of team working practices [Evans 1990]. The effect of such action is that design practicioners are faced with even greater volumes of product 
related data to digest and comprehend. As a consequence a requirement for the introduction of information technology systems has arisen [Kahaner and Lu 1993].

A dissatisfaction with the functionality of existing information technology (IT) tools has led to the current interest in SE support. However, the development of complete information technology systems for the support of simultaneous engineering is a relatively young research field concerned primarily with the creation of computer systems that enable the effective sharing of information [Jagannathan et al. 1991]. A complete specification for such systems has not yet been universally agreed. Industrially oriented systems have often focused on developing applications to support one of the many specialist tasks that come under the simultaneous engineering banner, whilst research systems have tended to embrace the wider information structure and software architecture issues. This has led to a number of diverse IT systems being proposed as SE support tools [Molina et al. 1994a].

The research reported on here is related to the development of one system, MOSES, that aims to support SE by providing two key information sources, a product and manufacturing model, together with an architecture that enables dedicated application environments to be added onto the system as required. In order to classify how the MOSES system compares with others it is essential to be able to document what kind of understanding of SE is reflected in MOSES, to what extent MOSES represents an organisational perspective, how MOSES has been developed and how MOSES has been implemented.

The similarities and differences between the goals of different system developers can be highlighted by exploring these issues. The focus of the second part of the paper is on a CAE Reference Model that has been developed to address these issues. It is the authors' opinion that the Reference Model could provide a common basis for comparison and a useful guide for the development of CAE systems that support simultaneous engineering.

\section{THE MOSES CONCEPT}

The MOSES project is a joint undertaking between Loughborough and Leeds Universities and is funded by the EPSRC. The specification of the research into MOSES focused on a computer based system that provides product and manufacturing information, enables decision support based on these information sources and is co-ordinated in a manner that makes it suitable for operation in a simultaneous engineering environment [ACME/SERC 1991]. The architecture of the system is shown in Figure 1 and consists of two data models (Product and Manufacturing Models) linked, by an integration environment, to a number of application environments. An application called the Engineering Moderator ensures that the evolving product design considers the different life cycle activities that are represented by the application environments.

The operation of MOSES is such that any number of application environments may be supported. Application environments are sometimes referred to as 'Design For $\mathrm{X}$ ' applications. The configuration and functions of the selected application environments will depend on the needs of the host organisation e.g. a 'design for maintenance' application environment may be important to an automobile manufacture but not required by a disposable watch manufacturer. All product related information is stored within the product model as a design evolves. This is the sole consistent source of product information. Should an application be triggered, then it operates on product information from the product model and any product information that it generates is added to 
that model. The manufacturing model is the sole source for manufacturing information and hence all applications obtain their manufacturing information from it [Ellis et al. 1993a] . The user may trigger any of the application environments. Conversely they may also be triggered by the Engineering Moderator. The Moderator monitors the product model to ensure that conflicts in information requirements are identified.

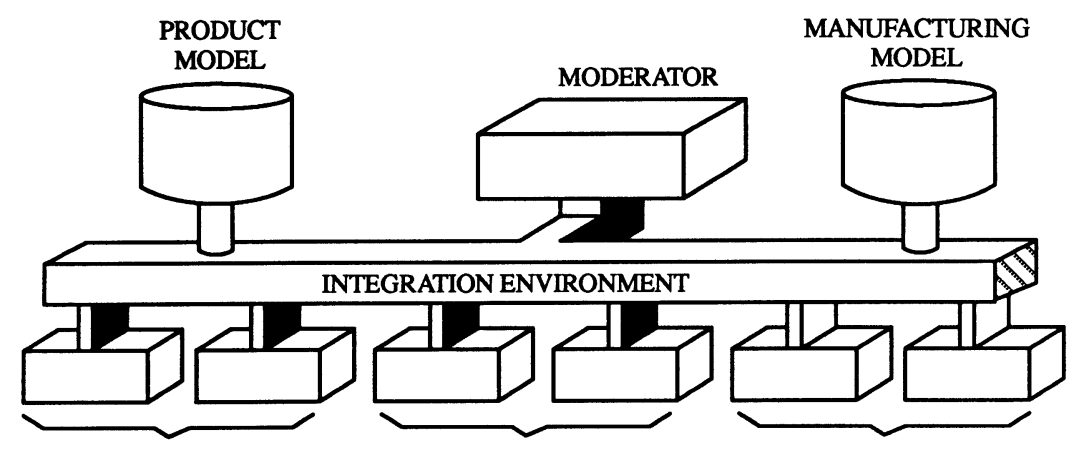

APPLICATION ENVIRONMENTS

Figure 1 The Structure of the Model Oriented Simultaneous Engineering System (MOSES).

In developing the MOSES system it is intended that the information and procedures necessary to support that part of the simultaneous engineering process concerned with concurrent design for manufacture be identified, and that a prototype knowledge and software environment be used to demonstrate this. To this end an application environment for the support of design for manufacture is being developed. This makes extensive use of both product and manufacturing data and hence exercises and tests the validity of the data models. In order to ensure industrial relevance a total of eight collaborating organisations contribute to the project.

\subsection{Product model}

One of the data models key to the MOSES project is the Product Model (PM). The Product Model contains all data related to a products' life cycle and is based, wherever possible, on the evolving STEP standard [ISO CD 10303 - 1].

STEP ("Standard for the Exchange of Product Model Data") is the most significant effort towards the development of integrated product models is that currently being undertaken. This standard aims to define a neutral data format for the system independent representation and exchange of all product related life cycle data. The information scope and possible forms of implementation covered by STEP go far further than previous interchange formats such as IGES, SET and VDAFS which concentrated on representing geometric data [Bloor and Bowen 1991]. The aim is to define a standard that can be used as the basis for the development of application oriented software. The researchers on the project have contributed to the STEP standard and within the project several areas of product modelling that have yet to be addressed by the STEP community are being developed. In particular the representation of specifications and assembly relationships 
are being explored. The nature of the new'work on specification, assembly and planned process representations is outlined below.

The aim of the specification modelling research is to build representations that allow specification information to be available for use in other stages of the product development life-cycle [ Mckay \& Erens 1994]. An IDEF0 activity model for the process of product specification has been established and a preliminary specification data model has been documented and tested through the use of case study data in a prototype implementation. The work to date has addressed different aspects of specification such as product variants and techniques that allow product families to be described in a conceptually efficient way. The work to date has concentrated on mechanical products and further research is being undertaken to establish the relationships between original and variant product specifications.

The product data framework available from previous research and STEP considered assemblies as lists of parts without reference to their functional or physical connectivity. This is not sufficient for applications such as tolerance analysis and design for assembly that require information on the nature of relationships between components [Henson et al 1993]. The research undertaken as part of MOSES has concentrated on combining the existing product data model framework with other data structures to support information about assemblies. The information requirements of the assembly data model have been defined and representations for the function and behaviour of components in an assembly are being researched.

If a product model is to support all life cycle activities then the information associated with the manufacture of the product must be represented. The nature of simultaneous engineering is such that as the product design evolves its manufacturability must be considered. This involves associating the product with both manufacturing processes and resources. The STEP community has developed representations for Integrated Generic Resources [ISO CD 10303-49] and an application protocol for the Numerical Control Process Plans for Machined Parts [ISO CD 10303-213]. The research in MOSES is looking to see how this can be extended to enable the information associated with design for manufacture to be represented. The work undertaken by STEP to date is aimed at representing the information that is in a complete process plan. Because by nature design for manufacture takes place on incomplete product designs then the information structures necessary are currently insufficient. To date a schema for planned processes has been developed that allows elements of a products description to be associated with particular manufacturing processes and activities. The resources used to perform each activity, and any manufacturing advice to be associated to any of these elements, can be modelled.

\subsection{Manufacturing model}

The second key data model within the MOSES system is the Manufacturing Model. It was found in previous projects that many applications require access to a consistent source of manufacturing information. Often this information is stored in different application systems and databases. In this scenario, data duplication can therefore be a problem. A goal of the MOSES project was to formulate the scope and logical structure of a Manufacturing Model that would integrate, and store without redundancy, the information contained in these separate sources.

The basis for the research into this type of data model came from pioneering work done during the IMPPACT project on a Factory Model [IMPPACT Esprit No. 2165]. Subsequent work on a Manufacturing Resource Model [Kimura 1991], Facility Model [Molina et al. 1992] and Manu- 
facturing Model [Al-Ashaab and Young 1992] have helped shape the nature of the MOSES Manufacturing Model. This project collaborates with other standardisation bodies (e.g. Project 2 of the ISO/TC184/SC4/WG8) to develop an application oriented representation of Resource Usage Management information and function.

The Manufacturing Model describes and captures the information about the manufacturing situation of a company in terms of its manufacturing facility and capabilities at different levels of abstraction [Molina et al. 1994b]. Three entities can be regarded to be basic elements in the definition of any manufacturing environment: resources, processes and strategies. These entities are relevant and important for any type of manufacturing firm. The relations and interaction among them builds the manufacturing environment of a company. It is these three elements that the MOSES Manufacturing Model represents.

Manufacturing resources are all the physical elements within a facility that enable product manufacture e.g. production machinery, production tools, material handling equipment, storage systems etc. The resources are often organized into groups to create manufacturing facilities such as stations, cells or shops. Resources have to be represented in a function oriented manner in order to describe their role in supporting the design, manufacture and production activities. A description of the resources based on their physical properties and functional composition allows the capture of their capabilities. Being able to represent resource capability enables the support of design decisions (e.g. design for manufacture) and manufacturing functions (e.g. process planning). A complete schema for resources has been developed, however, only that part associated with chip forming machinery has been fully defined and implemented.

Manufacturing processes are those processes carried out in a facility in order to produce a product. There are in general two types of manufacturing processes, information and material processes. If a CNC machine is producing a component and the cutting tool breaks then the material processes terminates, however, the information process continues as the $\mathrm{NC}$ programme continues to be processed. To limit the scope of the model it was decided from the outset to consider only the machining and injection moulding processes. The Manufacturing Model contains associations between processes and resources. There are many alternative resource combinations that can be employed to perform a given process. It is the role of an external application to select from the alternatives based on a knowledge of the product. It is vital that the content of the Manufacturing Model remain non-product specific if its use is to be maximised.

The representation of structured resources and processes allows us to have a reliable representation of the manufacturing facilities and their capabilities in terms of process technology and equipment. In addition to this type of information, there is a need to represent the manufacturing strategies. Strategies are decisions made on the use and the organization of resources and processes. In effect they are the constraints imposed on the use of a certain type of resource or process that differentiate an organisations manufacturing operation from that of others. There are two types of decisions which make possible the formulation of manufacturing strategies: decisions made over time which define the structure, capacity and technology of the facilities, and the day to day decisions which determine how to use the facilities and related processes. In the Manufacturing Model, strategies refer to capacity, facility structure, technology (i.e resources and processes), production planning/material control and the organization of work. 
The Manufacturing Model has been structured into four levels based on a defacto standard [BSI PD 6526:1990]:

1. Factory Level

2. Shop Level

3. Cell Level

4. Station Level.

These levels of abstraction provide manufacturing information for all hierarchical and functional activities within a manufacturing enterprise. For the data model to be of practical use it must reflect at any given instant the actual resources in the factory. A prototype Manufacturing Model has been developed that represents the manufacturing capability of a collaborators machine shop (Figure 2). The manufacturing model has been documented in EXPRESS [ISO CD 10303 - 11] to ensure consistency with the practices of other ISO standardization groups.

\subsection{Engineering moderator}

$S E$ requires that a number of different tasks be inter-woven within a given time period. Each task has its own goals and a hierarchy is imposed that allows trade-offs between goals at different levels, in effect some tasks are given higher priority. The nature of the tasks is such that they can require pre and post actions. SE is therefore often considered to consist of a number of parallel tasks but inevitably there are some critical paths which impose a sequence on events.

Research into computer support for SE has often concentrated on trying to resolve this complex inter-relation of tasks. This has proved difficult because SE rarely involves straightforward 'individual' actions. Apparently discrete activities rely upon a complex network of factors and systems to date do not adequately deal with this interaction.

The MOSES approach to imposing a measure of concurrency on the operation of the system is to use an application called the Engineering Moderator. The aim of the Moderator is fourfold [Harding 1993] :

1. Promote co-operation and negotiation between SE team members

2. Identify significant problems within a design

3. Determine appropriate action in response to a problem

4. Maintain communication between interested agents until the problem is resolved

The Moderator bases its operation on assessing the product information currently available rather than on monitoring a sequence of tasks. As a design evolves the data related to the product is entered into the product model. The product model is therefore the most reliable indicator of the state of the current design. The moderator works by monitoring and analysing the proposed input to the product model in order to assess whether the user, or any of the application environments, should be advised of the design change. To achieve this the moderator has an inbuilt knowledge base which contains details of the types of product information change which are particularly sensitive to each application environment. 
All the application environments access shared product information in the product data model. This leads to situations were tasks may be disrupted due to a mismatch of expectations. For example if a design is being analysed for design for manufacture then a design change may be recommended that conflicts with that of the design for function requirements. If undetected then inappropriate actions may be carried out. However, the moderator attempts to get the applications to realign their expectations. The moderator needs to ensure the timeliness of its intervention and the authors believe this is best achieved by being aware of changes to the product data model as they occur.

In effect the moderator enables the system to become self-organising because the way in which the system responds depends on the way in which the PM evolves. There is no fixed procedural task structure for the design process. Hopefully this improves the competence of the system and supports interaction and conversation because each application is basing its decision to act on the information available to it rather than on a false preconceived model of design tasks.

The sensitivity of the Moderaotor to product information change is critical because, if incorrect, it is possible that some applications could be continuously switched between tasks. A simplified prototype Engineering Moderator has been developed that successfully monitors product model changes and subsequently triggers the relevant application.

\subsection{Application environments}

SE systems are still often compartmentalised into predominantly problem centred DFX application groups. This is because it enables researchers and developers to deal with smaller information sets and also because research is often theoretically driven and involved in resolving highly specialised research topics. Rather than replace this view the authors believe it can prove an advantage if the information sources used by each application group are common, and that the working of each is co-ordinated. It is essential that future CAE systems be extensible. By modularising the application groups that use the services of the data models this becomes feasible.

The resources available to the project have necessitated that only one application environment, addressing design for manufacture, be fully researched [Ellis et al.1993b]. This shall be referred to as the Design For Manufacture Environment (DFME). An application environment concerned with design for function is being developed in research related to the project . Design for manufacture was selected because it is an application area that can exercise most elements of the MOSES system. The structure of information in the product and manufacturing models can be tested and the operation of the moderator investigated.

Design for manufacture (DFM) is one of many facets of simultaneous engineering and aims to support experienced designers by helping to ensure that certain key design rules are systematically addressed. The design rules help ensure that the product design can be manufactured with the processes and resources available to the organisation.

The DFME aims to provide reliable, and timely, enterprise specific design for manufacture advice to the user and must ultimately also support the generation of manufacturing information e.g. process planning. To perform design for manufacture on an evolving product design it is necessary to analyse the component in relation to process capability and available resources. This information is stored in the product model because it is of relevance to later activities such as producing process plans. Indeed the nature of post design manufacturing activities such as process 
planning and scheduling could be significantly changed by undertaking DFM using such a CAE support system.

Though the actions of the design for manufacture environment are determined by the amount of information available in the data models, it is useful to relate this to some traditional design model in order to show the type of activities undertaken. Elements of DFM are applied throughout the product design process. This is assumed to consist of conceptual, embodiment and detailed design phases [Pahl and Beitz 1984]. At the conceptual stage designers often assume a particular manufacturing method and are interested only in the general capability of processes e.g. material suitability, product size, estimated tolerance bands. At the embodiment stage the design team requires information on the capabilities of various processes e.g. form constraints, resource types, workholding methods. In the detailed design stage they will benefit from information related to the potential capabilities and availability of specific machines and tools and the way in which the combination of these effect geometric, surface and dimensional tolerances. We therefore propose that there are three query types that the DFME environment must be able to respond to:

1. Product suitability for manufacture by a given process

2. Product suitability for manufacture using a given resource type

3. Product suitability for manufacture using a specific resource combination.

Process suitability queries will prompt the DFME to determine which manufacturing processes are capable of manufacturing a given product, for a given volume, material and approximate shape. Resource Type suitability queries prompt the DFME to determine how suitable a component, or a feature of a component, is for manufacture by a given process and workstation type. Specific resource suitability queries prompt the DFME to determine how suitable a particular component, or component feature, is for being produced using a named resource, or resource set, that is available to the Enterprise. The nature of feedback to the user and product model is to list the feasible solutions and to comment on the implications of product design in terms of cost, lead time and quality. The DFM analysis considers the product specification, form and tolerances. It is assumed that the specification contains manufacturing due date, volumes and required batch sizes.

The DFME is partitioned in such a way that it has one governing application called an manufacturing strategist. The role of the strategist is such that it governs a hierarchy of application subgroups. In the DFME a range of process groups are represented e.g. machining and injection moulding. In turn each of these has sub-experts e.g. machining would consist of experts for turning, milling etc. Each application group and expert models a set of constraints related to their particular subject area. The Strategist must determine which of these sub-groups to trigger based on the available product information. It may be that several sub-groups are activated. In this case the Strategist must evaluate between the proposed feasible alternatives returned by the subgroups. The Strategist also overseas the requests from applications for information from data models and the users. The user may be asked to make selections in order to direct a search sequence or may have to provide additional product information in order to make a search feasible.

In essence the DFME contains mechanisms for making associations between product information and process/resource information. In order to demonstrate the principles involved in the research a much restricted implementation of the DFME is being developed that communicates 
with the product and manufacturing models. The role of the DFME is being documented using the Booch methodology [Booch 1991] and IDEF0 diagrams [Colquhoun et al. 1993].

\section{THE CAE REFERENCE MODEL}

The activity of co-ordinating and structuring complex CAE systems during design and development necessitates the use of formal methods. The MOSES research is being guided by the use of a CAE Reference Model (CAE-RM) to provide a framework for the integration of modelling methodologies and computer tools [Molina et al. 1994c].

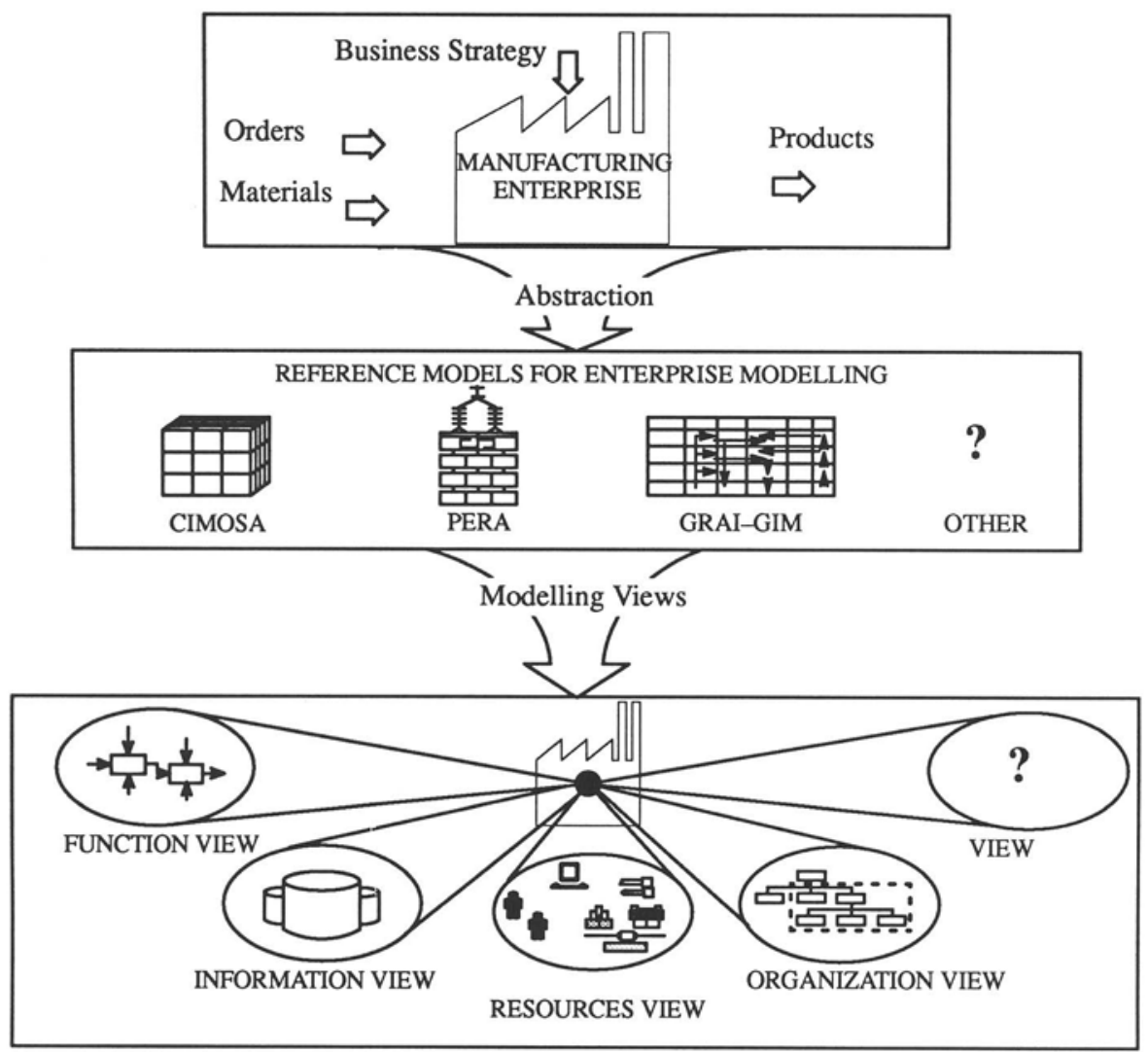

Figure 2. Reference Models for Enterprise Integration and related Modelling Views

The Booch Object Oriented Methodology [Booch 1991], IDEF0 [Colquhoun et al. 1993] and EXPRESS [ISO CD 10303 - 11] are methodologies that are specified within the CAE-RM to assist in the modelling of different aspects of a system. A number of supporting computer tools have been used to maximise the effectiveness of these methodologies. They include STEP 
Programmer's Tool Kit (STEP Tools Inc. 1993) for compiling and refining EXPRESS schemas, and the RATIONAL ROSE (Rational 1993) software tool for Booch documentation.

Many current systems being developed to support SE are open in nature and distributed so as to allow remote operation and extensibility. The Reference Model for Open Distributed Processing or RM-ODP [SC21/WG7 N 755] is an emerging standard focused on open distributed systems. The CAE-RM that has been developed by the MOSES team is based on this reference model so as to enforce the generic and modular characteristic of the model.

The RM-ODP allows the description of an information system from different viewpoints: Enterprise, Information, Computation, Engineering and Technology. Each view represents a specific aspect of the information system. The views of the RM-ODP aim to allow a complex system to be described from a number of perspectives. The emphasis of each view is tailored to primarily represent either objectives, realisation or behavioural attributes. The research currently being undertaken is mainly involved with the definition of the first three viewpoints i.e. Enterprise, Information and Computation.

The Enterprise Viewpoint is associated with the specification of requirements for ODP systems. IDEF0 activity diagrams are employed to describe this view. The information viewpoint is particularly important to the development of MOSES since this sets the context for the development of the information models in an implementation independent form. The information flows, together with information structures, are represented at the Information Viewpoint. This view is documented using EXPRESS and IDEF0 models. The Computational Viewpoint focuses on the functional decomposition of the system into objects, the activities that occur within those objects and the interactions between the objects. Booch's diagrams are used to describe this view.

The last two viewpoints (Engineering and Technology) have not been fully developed by the MOSES team. The Engineering Viewpoint focuses on the infrastructure required to support distribution. This view enables the specification of the processing, storage and communication functions required to implement the system. The Technological Viewpoint focuses on the selection of the necessary technology to support the system. In our case we chose the object oriented database DEC Object/DB [Objectivity/DB 1991] and the object oriented programming language $\mathrm{C}++$ [Stroustrup 1986].

A reference model should allow the scope of a system to be defined. SE systems are traditionally described in terms of their architectures and how to use themi.e. the use of formalised approaches to describe technology driven research. Whilst this is adequate for standalone systems, a vital element that is missing is how the system will support and be integrated in the organisation in a wider context.

A wide range of different reference models, frameworks and architectures exist for use in information system and manufacturing systems development. Among these reference models, the ones related to modelling and implementation of CIM systems can be used, in the opinion of the authors, to define a context for where future computer systems to support simultaneous engineering can be integrated within the enterprise. The reference models most often considered for the task of describing an integrated system, its life cycle and the methodology for its application are CIM-OSA [ESPRIT Project 688/5288], Purdue Enterprise Reference Architecture [Williams 1991] and GRAI-GIM [Doumeingts et al. 1992]. These reference models enable the creation of enterprise models that take into consideration different viewpoints (Figure 3). The authors believe that using these enterprise integration frameworks to set the context for the research into a Reference Model for Computer Aided Support of Simultaneous Engineering allows the cre- 
ation of a more structured and flexible model. The MOSES CAE-RM achieves this, and ensures integration within a wider framework, by defining a mapping to the CIM-OSA architecture. The CIM-OSA architecture is such that a reference model for a technical support system, such as a CAE system, can be defined as a resource within the architecture. This is achieved by defining the inputs, outputs and required capabilities of the technical system. The information and function elements of the architecture set the context for the requirements and the use of that resource i.e. the CAE system (Figure 4).

\section{DISCUSSION}

The development of effective information structures for the representation of both product and manufacturing information will largely determine the success of CAE support for simultanoeus engineering. There is considerable research into product modelling and some of the work generated by the STEP community is being adopted by system vendors and hence slowly permeating into industry. The scope for development of the STEP standard is vast. Within the MOSES project the research into representing specification and assembly relationships should improve product model utility.

Research into manufacturing modelling has not received as much attention, however, the indicators are that this is a rapidly developing field. Several groups are focusing their effort on developing virtual factories and enterprise models. To support this concept the manufacturing models of the future will have to represent not only manufacturing capability and characteristics but also they will have to model the dynamic nature of a facility. This will enable them to support simulation and scheduling applications.

An important element of the MOSES Manufacturing Model is the ability to model strategies for the use of processes and resources. The way in which an enterprise operates is determined not only by the technology available but also by the rules and standards that have developed with the company. Modelling strategies is one way of ensuring that company differentiation is not lost by the implementation of IT support. It also potentially allows the effect of changing manufacturing strategies to be studied.

The element that distinguishes CAE support for simultaneous engineering from that of conventional CAE support is the management of the complex interaction of varied tasks. The MOSES project have chosen to use an application called the Engineering Moderator to enforce concurrency. This application bases its decision to act on the information content of the product model. Each addition to the product model is analysed and assessed to determine if either the user or application environments are likely to be interested in the change. Should this be the case then the relevant application or user is informed. Care is needed to ensure that the Moderator is not over-sensitive to change and hence constantly triggering actions. The advantages of this approach is that a preconceived design model is not followed and the evolving design is forced to consider all the life cycle activities represented by application environments.

The use of a CAE Reference Model to guide the design and development of the CAE system has proved valuable. The ability to represent different viewpoints of the same system is a major advantage. The RM-ODP provides a structured and well documented format for achieving this. The use of advanced computer tools which support modelling methods such as Rational Rose for Booch and STEP Tool Kit for EXPRESS accelerates the implementation of prototype software to prove system concepts. 


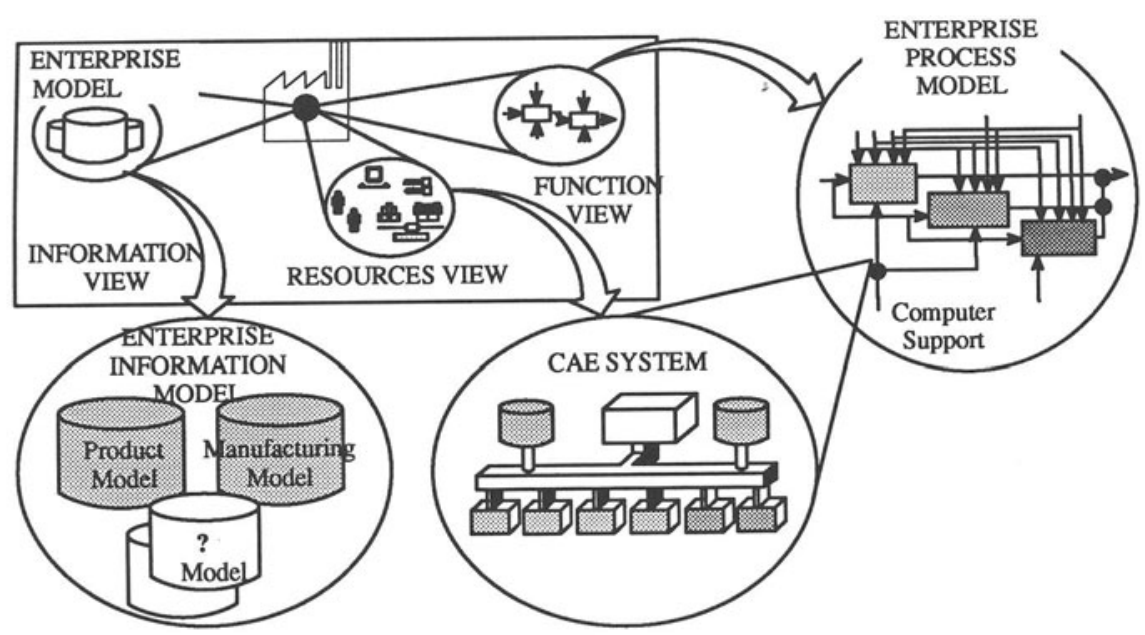

Figure 3. CIMOSA Enterprise Model as a Context for the MOSES CAE Reference Model and CAE System

The mapping of the CAE-RM to CIM-OSA has the advantage of setting IT support for SE in the context of an enterprise model. Potentially the influence of the system on existing working practices can then be modelled. The management of different perspectives in a company is often based on the strengths of the personalities involved and on the company culture [McCarthy 1994]. If IT support gives an equal status to each perspective then the nature of the design task in that company may be imbalanced and the design differentiation imposed by the company culture is lost. For this reason the system must be able to be customised to suit the organisation. It is only by studying this wider context that the impact of new technolgy can be predicted. Traditionally CADCAM has been a sequential development with technology considered before its effects on organisational and human aspects. The use of combined enterprise and CAE Reference Models should enable the selection of the most appropriate technology for the business. This process is lead by the need to achive complete enterprise integration.

\section{CONCLUSIONS}

The need to establish or sustain competitive advantage invariably imposes a requirement to introduce new information technology systems in virtually all contempory organisations at some stage in their development [Helliwell 1994]. Simultaneous engineering implementations have reached the level of maturity were organisations feel that they could benefit from more effective IT support.

The authors consider that the key issues in the CAE support of simultaneous engineering are information modelling, decision support and task co-ordination. The MOSES project has addressed these issues by:

- Developing Product and Manufacturing Information Models 
- Creating an environment for decision support

- Investigating mechanisms for task co-ordination

The complexity of developing such systems imposes a requirement for an integrated and formal approach to system development. This has been achieved within the MOSES project by defining and using a CAE Reference Model. The authors' experiences demonstrate that the use of a CAE Reference Model is necessary for effective system development. The benefit of the CAE-RM has been enhanced by building a mapping to the CIM-OSA framework. This ensures that the CAE system meets the requirements imposed by the concept of an integrated enterprise.

\section{ACKNOWLEDGEMENTS}

The research reported on in this paper is that undertaken by the whole MOSES project team and not solely that of the authors. The research is part of an ACME funded project, 'Exploiting Product and Manufacturing Models in Simultaneous Engineering', pursued at Loughborough University of Technology and Leeds University, and supported by a group of industrial collaborators [SERC ref. GR/H 24273 and GR/H 24266 respectively].

The research undertaken by A. Molina is funded by the Mexican Government (CONACyT), Monterrey Institute of Technology (ITESM, Mexico) and the ORS Award Scheme (ORS/9226010).

\section{REFERENCES}

ACME/SERC Proposal, 1991, Exploiting Product and Manufacturing Models in Simultaneous Engineering, Department of Mechanical Engineering-University of Leeds, Department of Manufacturing Engineering-Loughborough University of Technology.

Al-Ashaab A., and Young R.I.M., 1992, "Information Models: An Aid to Concurrency in Injection Moulded Products Design", Presented in the Winter Annual Meeting '92 of ASME. Anaheim, California, November. 8-13, 1992.

Bloor M.S., and Owen J., 1991, "CAD/CAM product-data exchange: the next step", Computer Aided Design, Vol. 23, No. 4, May, pp. 237-242.

Booch G., 1991, Object-orienied design with applications, The Benjamin/Cummings Publishing Company, Inc..

BSI PD 6526:1990, CEN/CENELEC Report R-IT-01, Evaluation on CIM architectures, British Standard Institution.

Colquhoun, G., Baines, R.W., and Crossley R., 1993, "A state of the art review of IDEF0", Int.J. Computer Integrated Manufacturing, Vol. 6, No. 4, 252-264.

Corrigall M.J., Lee M.K., Young R.I.M., and Bell R., 1992, "Manufacturing Code Generation in a Product Modelling Environment”, Proc. Instn Mech. Engrs., Vol. 206, pp. 165-175. 
Doumeingts G., Vallespir B., Darricar D., and Chen D., 1992, GIM, GRAI Integrated Methodology, A Methodology for Designing CIM Systems, Version 1.0, Unnumbered Report, LAP/GRAI, University Bordeaux 1, Bordeaux, France, May.

Ellis, T.IA., Young R.I.M., and Bell R., (1993a). Modelling Manufacturing Process Information to Support Simultaneous Engineering. Proceedings of the ICED 93, Volume 2, N.F.M. Roozenburg (ed.), 9th International Conference of Engineering Design, August 17-19, 1993, The Hague, pp. 1081-1084.

Ellis, T.IA., Young R.I.M., and Bell R., (1993b). A Design For Manufacture Software Environment to Support Simultaneous Engineering. Proceedings of the 9th National Conference on Manufacturing Research, Alan Bramley and Tony Mileham, September 7-9, 1993, University of Bath, U.K. pp. 1081-1084.

ESPRIT Consortium AMICE, 1993, CIMOSA: Open System Architecture for CIM, Project 688/5288, AMICE, Volume 1, 2nd revised and extended edition, Springer-Verlag.

ESPRIT Project 688/5288, AMICE, Volume 1, CIMOSA: Open System Architecture for CIM, ESPRIT Consortium AMICE (Eds.), 2nd, revised and extended edition, Springer-Verlag, 1993.

Evans S., 1990, "Implementation framework for Integrated design teams", Journal of Engineering Design, 1(4), pp. 355-363.

Harding J.A. , 1993, The Engineering Moderator Within The MOSES Project, MOSES discussion document, moses-dd-21, 29/3/1993.

Helliwell G., 1994, Introducing IT into a Mature Production Related WOrk Environment: The Human Resource Factor, Journal of Information Technology, Vol.9 No.1, March 1994, pp. 39-50

Henson B.W. , Baxter J.E. , Juster N.P. , 1993, Assembly Representation Within a Product Data Framework, Proceedings of ASME Design Technical Conferences, USA, September 1993.

IMPPACT, Esprit No. 2165, Proceedings of the Workshop Integrated Modelling of Products and Process using Advanced Computer Technologies, Berlin, 26-27 February 1991.

ISO CD 10303 - 1, 1992, Product Data Representation and Exchange - Part 1: Overview and Fundamental Principles.

ISO TC184 SC4 WG8/N13, Project 2: "Resource Usage Management", Draft Scope presented at the London Meeting by B.R. Katzy, June, 1992.

ISO CD 10303-213, Numerical Control (NC) Process Plans for Machined Parts version 1.0, $1 / 5 / 1994$.

ISO CD 10303-49, Integrated Generic Resources: Process Structure and Properties,

ISO CD 10303 - 11, 1993, Industrial automation systems and integration-Product data representation and exchange-Part 11: Description methods: The EXPRESS language reference manual.

Jagannathan V., Cleetus K.J., Kannan R., Matsumoto A.S., and Lewis J.W., 1991, "Computer Support for Concurrent Engineering", CONCURRENT ENGINEERING Issues, Technology and Practice, September, pp. 14-30. 
Kahaner D., and Lu S., 1993, 'First CIRP International Workshop on Concurrent Engineering for Product Realization", Concurrent Engineering Research in Review, Volume 5, pp. 6-14.

Kimura F., 1991, "Software for Product Realization", International Symposium for International Trends in Manufacturing Towards the 21st Century, October 18, Berlin, Germany.

McCarthy J., 1994, 'The State-of-the-Art of CSCW: CSCW Systems, Cooperative Work and Organisation”, Journal of Information Technology, Vol. 9 No. 2, June 1994, pp. 73-83

Mckay A. , Erens F., Relating Product Specifications and Product Definitions in a Product Model, Moses report series, moses-report-series-1, 14/1/1994.

Molina A., Mezgar I. , and Kovacs G., 1992, "Object Knowledge Representation Models for Concurrent Design of FMS", Human Aspects in Computer Integrated Manufacturing, G.J. Olling, F. Kimura (editors), Elsevier Science Publishers B.V., (North-Holland), IFIP, pp. 779-788.

Molina A., Al-Ashaab A.H., Ellis T.I.A, Young R.I.M, Bell R., 1994a, "A Review of Computer Aided Simultaneous Engineering Systems", Accepted for publication in the journal of Research in Engineering Design, June 1994.

Molina A., Ellis T.I.A, Young R.I.M, Bell R., 1994b, "Modelling Manufacturing Resources, Processes and Strategies to Support Concurrent Engineering", to be presented in the First International Conference on Concurrent Engineering: Research and Applications, August 29-31, 1994, Pittsburgh, PA, USA.

Molina A., Ellis T.IA., Young R.I.M., and Bell R., 1994c, "Methods and Tools for Modelling Manufacturing Information to Support Simultaneous Engineering", Preprints, 2nd IFAC/IFIP/ IFORS Workshop Intelligent Manufacturing Systems - IMS'94, (Ed. P. Kopacek), Vienna - Austria - June 13-15, 1994, pp. 87-93.

Nevins J.L. and Whitney D.E., 1989, Concurrent Design of Product and Processes: A Strategy for the Next Generation in Manufacturing, McGraw-Hill Publishing Company, N.Y.

Objectivity/DB, 1991, Reference Manuals, Objectivity Inc.

Pahl G., and Beitz W., 1984, Engineering Design: A Systematic Approach, Springer-Verlag.

Rational Object Oriented Software Engineering, 1993, RATIONAL ROSE ANALYSIS \& DESIGN, SUN PLATFORM, Version 1.1.

SC21/WG7 N 755, 1993, Draft Recommendation X.901: Basic Reference Model of Open Distributed Processing - Part 1: Overview and guide to use, ISO/IEC JTC1/SC21/WG7.

Stroustrup B., 1986, The C++ Programming Language, Second Edition, Addison-Wesley. STEP Tools Inc., 1993, STEP Utilities Reference Manual, Version 1.2 for UNIX Workstations. Williams T.J., 1991, The Purdue Enterprise Reference Architecture, Report Number 154, Purdue Laboratory for Applied Industrial Control, December.

Young, R.I.M. and Bell R., 1992, Machine Operation Planning in Product Modelling Environment, International Journal of Production Research, Vol. 30, No. 11, pp. 2487-2513. 